\title{
Face recognition attendance system using Local Binary Pattern (LBP)
}

\author{
Shamsul J. Elias, Shahirah Mohamed Hatim², Nur Anisah Hassan ${ }^{3}$, Lily Marlia Abd Latif ${ }^{4}$, R. \\ Badlishah Ahmad ${ }^{5}$, Mohamad Yusof Darus ${ }^{6}$, Ahmad Zambri Shahuddin ${ }^{7}$ \\ ${ }^{1}$ Universiti Teknologi MARA (UiTM), Malaysia \\ 2,3,4,6,7 Faculty of Computer and Mathematical Sciences, Universti Teknologi MARA, Malaysia \\ ${ }^{5}$ Faculty of Informatics and Computing, Universiti Sultan Zainal Abidin (UniSZA), Malaysia
}

\begin{tabular}{|c|c|}
\hline Article Info & ABSTRACT \\
\hline Article history: & Attendance is important for university students. However, generic way of \\
\hline Received Sep 30, 2018 & $\begin{array}{l}\text { taking attendance in universities may include various problems. Hence, a } \\
\text { face recognition system for attendance taking is one way to combat the }\end{array}$ \\
\hline Revised Nov 15, 2018 & problem. This paper will present an automated system that will automatically \\
\hline Accepted Dec 21, 2018 & saves student's attendance into the database using face recognition method. \\
\hline Keywords: & $\begin{array}{l}\text { face detection and face recognition. The face detection part will be done by } \\
\text { using viola-jones algorithm method while the face recognition part will be }\end{array}$ \\
\hline Face detection & $\begin{array}{l}\text { carried on by using local binary pattern (LBP) method. The system will } \\
\text { ensure that the attendance taking process will be faster and more accurate. }\end{array}$ \\
\hline
\end{tabular}

Copyright $@ 2019$ Institute of Advanced Engineering and Science. All rights reserved.

\section{Corresponding Author:}

Shahirah Mohamed Hatim,

Faculty of Computer and Mathematical Sciences,

Universti Teknologi MARA,

35400 Tapah, Malaysia.

Email: shahirah88@uitm.edu.my

\section{INTRODUCTION}

In today's world, attendance taking is essential. Attendance would be counted as a part of student's mark in most universities. Usually, attendance is taken by using a piece of paper that contains student's information and act as student's attendance register. Students will then put on their signature as a sign that they are present on that particular day.

However, there are problem with this generic technique. Some of the problem includes student's forging signature of their close friend [1]. The attendance process takes a long time and many more problems [2]. To counter these problems, an automated attendance system is needed to be done. In this paper, the project will present the automated system which is face recognition in order to combat these problems. It is an automated system that will save student's attendance automatically into the database.

In order to automatically take student's attendance, there are technologies that are readily built such as Barcode Readers, Radio Frequency Identification System (RFID) and Bluetooth. Even though these technologies may help to reduce the time taken for the attendance to be taken, it requires machine and devices that are expensive and have limited usage as the device is easily broken [3].

There is also other method such as biometrics. However, biometrics application proposal mostly involves the application to be used by the lecturer during class hour. This will eventually cause distraction as the class will be interrupted every time the lecturer allows late students into the class [4]. Using biometrics, it also will take a longer time as students need to queue in order to use the device [5]. 
Face recognition system is a perfect way to solve these problems. Using face detection, student face will be taken real time while the student is learning in class without them noticing and the learning process will be smooth. Students also can be attentive without being disturbed in order to sign for their attendance and lost some of the information that are given by the lecturer. As for the lecturer, there is no hassle in order to keep all the student's attendance registers for reports later because the attendance is automatically generated by the system. The data generated for the report will be accurate and there will be no more misleading data since the chances for students to forge the documents is eliminated.

Face recognition has been used for a long time in many fields. In today's world, face recognition can easily be done since cameras are readily and easily available in the market. Face images can easily be captured in the daily life as there were rapid development of electric equipment such as surveillance camera and cell phones [6].

One of the areas that face recognition plays important role in is security. In today's world, many premises such as houses use face recognition in order to enhance their house security. There are a face detection and recognition system for Smart Home Security Application designed by Ana and Wati [7] in order to fulfill the needs of home owners. It uses a directed face to decide whether or not a person is allowed into the premises. It has a high accuracy of face recognition.

Other study is a study done on automated face recognition of rhesus macaques by Witham [8]. This study is advantageous since it non-invasively identify rhesus macaques in videos to find out their group and lastly, there is an application software called Doggelgangger [9] that is developed to compare and match a canine with their possible human parents. This application shows that face recognition can also be used in various applications.

\section{ATTENDANCE SYSTEM}

The word student can be defined as "a person who is studying at a school, college, or university" according to Oxford Advance Learner's Dictionary, while the word attendance is defined as "the act of being present at a place" [10]. Referring to the definition earlier, student's attendance system can be considered as a system that are used in school, college or universities in order to mark or specify that a student is present or absent on a particular day.

The general way of attendance taking is usually done by using a piece of paper that has student's information. This paper will act as registry and will be passed around the classroom [11]. For example, the date on the registry will be filled up by the lecturer and the paper will be passing through the class. Sometimes, the lecturer will let the paper be passed while they start teaching in order to save time. Another way of taking attendance is lecturers will call the student's name one by one. If they are present, the students need to answer loudly enough for the lecturers or teacher to hear.

Both methods mentioned above have been practiced globally for a very long period of time. However, problem often arise when the normal or traditional attendance taking method are being used. They can be time consuming especially when students need to response when their names are being called by the lecturer [12]. The attendance report also will be hard to maintain until it is needed to generate the report as the attendance sheet or registry could have gone missing. Using the traditional method, teachers or lecturers also required to correctly mark the student's attendance [13], but then mistakes might happen while marking the attendance. If these occurs, the data can be affected when report are being generated.

Another problem that can be found when using the general method of attendance taking is students can deceive their lecturer by signing for their friends who is absent for the day [11]. This action can easily be performed especially in a big hall where it is impossible for the lecturer to count the number of students. In order to combat all these problem, the project propose an automated system using face recognition to automatically collect and save student's attendance.

\section{IMAGE PROCESSING}

Images can be taken from various sources. Some of the sources include cameras or sensors that are placed on satellites, space probes and even air crafts [14]. The image that are produced by these sources are called raw image. Image processing is a technique used to improve these raw images [15]. It can also be used with normal images that are taken for many activities in our everyday life [16]. Image processing, is a form of processing with image as its input and will produce characteristic or parameters that are related to the input image as its output [17]. There are numerous field and areas that can be involve with image processing [16]. The areas and fields include remote sensing, medical imaging, textile and several others. In image processing, there are three steps involved [15]. First step is importing the image using optical scanner or 
digital photography. The next steps are to analyses and manipulate the image and the final step is to get the output.

There are two different types of image processing. The first type is analog image processing and the other is digital image processing. Analog image processing is when the image is altered through electrical medium. A television image, adjusting the amplitude and reference of the video signal will result in the brightening, darkening and alteration of the brightness range of the displayed image [16]. Different in analog signal, digital image is being processed in two-dimensional image by using digital computer. A finite number of bits which is actually an array of the actual number represent a digital image.

\subsection{Segmentation}

Segmentation process can be defined as a process where image is subdivided into some components parts or object [18]. The subdivision of the level will be carried is depending on how the problem is being solved [14]. For example, we are interested in identifying a person in an alley. We need to segment the alley first from the image. Then, the content of the alley will be segmented to find the potential person. For image segmentation, image thresholding techniques are used.

There are two categories in image segmentation which s Layer-Based Segmentation and Block-Based Segmentation. In Layer-Based Segmentation, Layered Model is used in order to detect object and define shape and mask. The appearance and depth ordering also will be explained. Class and instance segmentation also will be evaluated by using image segmentation that composite the output of a bank of object detectors. Block-Based segmentation method on the other hand is based on various features that can be found in image. These features might be color information that is used to create histograms and could be the information about the pixels that could indicate edges or boundary. The features also could be the texture information [18]. Maintaining the Integrity of the Specifications.

\section{FACE RECOGNITION}

Face recognition can be referred as a process that requires facial images to be captured using a camera and saving it automatically into the database [12]. Face recognition, generally are divided into two stages. The first stage is face detection and the second stage is face recognition.

\subsection{Face detection}

In order to perform face recognition, the image captured must undergo face detection process first. Face recognition is a process where human face image is detected using algorithms that are associated with face detection. In face detection, there are many algorithms that are mostly used. Some of them are Ada Boost Algorithm, S-Ada Boost Algorithm Support Vector Machines (SVM) and Float Boost Algorithm. There are also other algorithms such as Bayes Classifier, HAAR Classifier and Viola Jones Algorithm [19].

Among the algorithms that are stated, Viola Jones Algorithm is said to have a low false detection a high accuracy compared to other method [20]. By using Viola Jones method, the features of integral image are computed and then classified. Instead of scaling the images, this method provides scaling of the features instead. Viola Jones method also said to be advantageous since the feature computations are extremely fast and it has efficient feature selection. Besides Viola Jones method, Ada Boost learning algorithm can also be used to choose the significant features from the computed potential features.

\subsection{Face recognition}

After key feature of an image has been extracted, it will be compared to the image that is readily stored in the database. This process is called face recognition process. In order to perform face recognition, there are few things that need to be taken into consideration. This is because there are a lot of issues that are associated with face recognition. Some of them are face pose, change in subject face such as eye glass, facial hair and veil for women [13]. There are also challenges such as illumination challenge when performing the face recognition in outdoor condition, face expression, face aging and dynamic background problems [21].

There are two parts of face recognition which is texture features and geometric feature [19]. Texture feature is when the face recognition is applied to some specific regions or the whole face while geometric feature includes parts such as eyes, nose, lips, eyebrows and other relation between those parts.

There are many types of technique and algorithm that can be use in order to perform face recognition. Some of them are Local Binary Pattern (LBP), Eigen face methodology and alignment-free partial face recognition algorithm [20]. Other methods that are available are Linear Discriminant Analysis (LDA), Kernel Method, Principal Component Analysis (PCA) and Neural Network [19]. Nevertheless, after comparing between PCA, LDA and LBP, it is said that LBP approach is the most effective algorithm [22]. Using LBP, a composition of small pattern will be formed by the facial image. The compositions are 
invariant to grey scale transformation. After the small patterns are combined, the whole description of facial image will be obtained.

\section{FLOW AND ALGORITHM PROCESS}

Figure 1 shows the project's architecture. The process will be initiated by image acquisition by camera of student's face image. The image then will be detected by using Viola-Jones algorithm. Then, the image will be compared to the image that are already stored in the database. The output of the comparison will be stored in the attendance database. Further explanation will be explained in step by step process below.

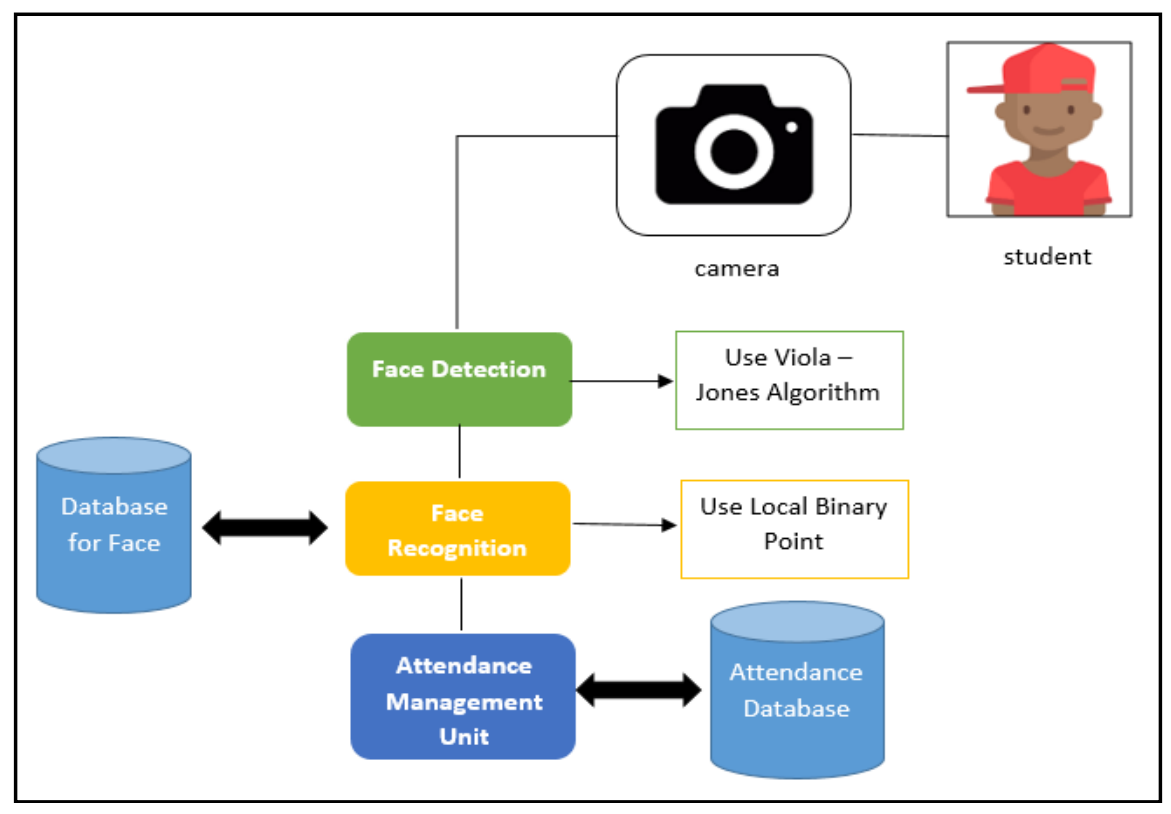

Figure 1. System architecture

\subsection{Image acquisition}

The initial step is the image will be captured from a camera that is positioned in the classroom. Ideally, the camera used must be a high quality camera as it is said that a high quality camera can produced much more features when being compared to a low quality camera. The camera can be positioned at the front of the class where it can capture the image of the whole class. The image should contain all the faces of students that are present in a particular class.

\subsection{Face detection}

In this research, face detection algorithm that will be used is Viola Jones Algorithm. In Viola Jones algorithm, there are four phases that are involved which is Haar feature selection, Integral image, Adaboost training and cascading [23].

In Haar Feature selection, the similarities of human face are represented by Haar feature. The features are represented by two or three rectangles that are composed together. For example, the human faces have similarities where normally, the nose bridge part will be brighter than the eyes. In this case, Haar features that represent the dark and white area are choosen. By using this technique, it is easier to detect whether face is present or not in an image.

Figure 2 shows the example of Haar feature. This Haar pattern usually can be obtain on the eyes part on human face. The bright part of the Haar feature represents human nose-bridge which is between the darker part of the Haar feature. In this figure, the darker part represents human eyes. 


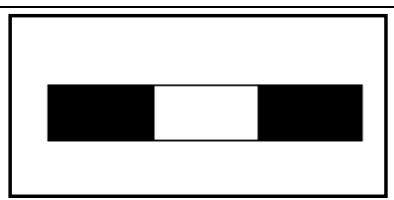

Figure 2. Example of haar feature

Each of the Haar features has their own value. The integral image is when the total summation of the pixel values from the original image is computed. The integral image is used in order to make a rapid computation of Haar features.

Since Viola Jones use a base window size of $24 \times 24$ window to evaluate the whole features in an image, there are about 160,000+ features that can be evaluated [12]. Hence, it is important to use Adaboost in order to select features that are useful and eliminate redundant features.

Finally, cascaded classifier classifies whether or not a sub-window is definitely not a face or it may be a face. If it is classified as may be a face, it will be passed to the next stage of the cascade, while if it is classified as not a face, it will be discarded.

\subsection{Face recognition}

Local Binary Pattern (LBP) will be used in order to perform face recognition. By thresholding the surrounding of each pixel with the value of the center pixel, LBP labels the image pixels. The result is considered as binary number [24].

When the bit pattern is circular and the binary pattern contains maximum of two bitwise transitions from 0 to 1 or the other way around, it is called uniform [25]. In LBP histogram computation, it uses uniform patterns so that the uniform and non-uniform pattern can be assigned to different bin.

\subsection{Comparison of features}

After the image has been through the face recognition stage, the features will be compared with the stored features. If there are similarities detected, it will be saved in the face database as well as the attendance database.

\subsection{Attendance database}

After the comparison has been completed, the attendance will be saved into the attendance database. If a student is present on a particular day, their student id will be saved together with the date and the course code of the class that the student participates in.

\section{RESULTS AND DISCUSSIONS}

As the project is a simple project, all the students that are already recognized will be marked their attendance that will be presented by Figure 3 .

After testing the system, the project finds out that under a controlled environment, the project shows a promising results. Some of the controlled environment includes:

a. Background.

In order for the face recognition to work at it best, the background need to be static. It is harder to recognize face in a dynamic background.

b. Lighting.

Lighting also impose an important aspect in recognizing face. An environment with adequate light shows more accurate results rather than a dim environment.

c. Facial changes.

Some changes to the facial area may cause inconsistency in recognizing the student's faces. For example, students with veil and spectacle are harder to be recognize if they did not wear their veil or spectacle.

Hence, it is important to address these issues in the future in order to enhance the accuracy of the face recognition process. 


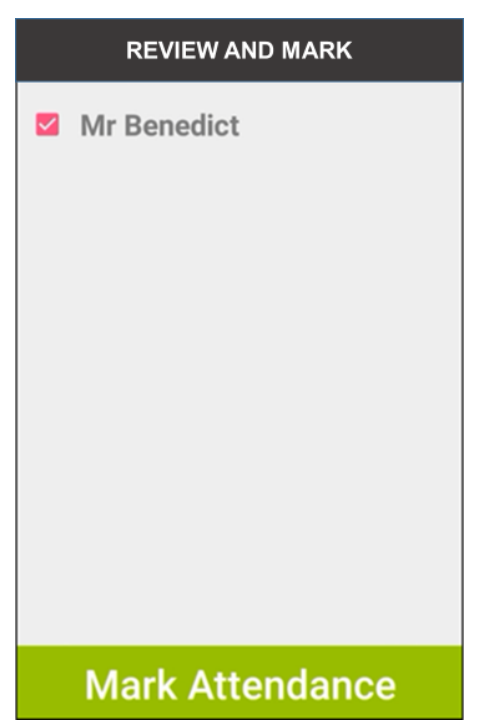

Figure 3. Attendance marking page

\section{CONCLUSION}

For the conclusion, this paper presents the automated system that will aid attendance taking process to be faster, more reliable and accurate. The project has already achieve all the traits that will make this project becomes reliable and useful to be used in order to take student's attendance. Using this system, the attendance taking process does not take a longer time compared to the traditional method. This system also assists the administration team by automatically save student's attendance. However, there are some issues that need to be address in the future. Some of them might include the background dynamics where the system can recognize face even though the background moves. Other issues will be the changes to the student's face and also the illumination of the environment where it should have enough light for the recognition to work. Hopefully, this research will satisfy the growing needs of technological advancement in attendance taking process since the traditional process of attendance taking have many flaws that need to be covered.

\section{REFERENCES}

[1] J. Chin, E. Tsun, C. W. Jen, F. Choong, and C. Mei, “Automated Attendance Capture System,” 2014.

[2] M. G. Krishnan, Balaji, and S. Babu, "Implementation of Automated Attendance System using Face Recognition," International Journal of Scientific \& Engineering Research. vol. 6, no. 3, pp. 30-33, 2015.

[3] U. A. Patel, "Computer Science and Management Studies Development of a Student Attendance Management System Using RFID and Face Recognition: A Review," Int. J. Adv Res., vol. 2, no. 8, pp. 109-119, 2014.

[4] F. Masalha, "A Students Attendance System Using QR Code," vol. 5, no. 3, pp. 75-79, 2014.

[5] S. Aiswarya, J. Peter, M. Cyriac, and L. S. Mathew, "Automated Attendance Management System using Face Recognition,” Int. J. Comput. Appl., no. ICWET 2015, pp. 10455-10457, 2015.

[6] Y. Li, W. Zheng, Z. Cui, and T. Zhang, "Face recognition based on recurrent regression neural network," Neurocomputing, vol. 297, pp. 50-58, 2018.

[7] D. A. R. Wati and D. Abadianto, "Design of face detection and recognition system for smart home security application," 2017 2nd International conferences on Information Technology, Information Systems and Electrical Engineering (ICITISEE), Yogyakarta, 2017, pp. 342-347.

[8] C. L. Witham, "Automated face recognition of rhesus macaques," J. Neurosci. Methods, vol. 300, pp. 157-165, 2017.

[9] "Doggelganger: Human to Canine Pairing Software-Dog Milk." [Online]. Available: dog-milk.com/doggelgangerhuman-to-canine-pairing-software/. [Accessed: 27-Apr-2018].

[10] Oxford Advanced Learner's Dictionary, 8th ed. 2011.

[11] S. A. M. Noor, N. Zaini, M. F. A. Latip and N. Hamzah, "Android-based attendance management system," 2015 IEEE Conference on Systems, Process and Control (ICSPC), Bandar Sunway, 2015, pp. 118-122.

[12] M. Shirodkar, “Automated Attendance Management System using Face Recognition,” no. Icwet, pp. 23-28, 2015.

[13] M. Fuzail et al., "Face Detection System for Attendance of C lass' Students," 2014.

[14] G. Padmaja and P. Nirupama, "Analysis of various image compression techniques," ARPN J. Sci., vol. 89, no. 86, pp. 86-89, 2017.

[15] P. M. Monga and S. A. Ghogare, "Scrutiny on Image Processing," Int. J. Adv. Res. Comput. Sci. Softw. Eng., vol. 5, no. 1, pp. 411-416, 2015. 
[16] S. Kaur et al., "Overview of Image Processing," International J. Adv. Res. Electron. CS Commun. Eng., vol. 4, no. 2, p. 190, 2015.

[17] S. Ojha and S. Sakhare, "Image processing techniques for object tracking in video surveillance- A survey," 2015 Int. Conf. Pervasive Comput., vol. 00, no. c, pp. 1-6, 2015.

[18] N. M. Zaitoun and M. J. Aqel, "Survey on Image Segmentation Techniques," Procedia Comput. Sci., vol. 65, no. Iccmit, pp. 797-806, 2015

[19] K. S. Selvi, P. Chitrakala, and A. A. Jenitha, "Face Recognition Based Attendance Marking System Abstract :".International Journal of Computer Science and Mobile Computing. 2014, vol. 3, no. 2, pp. 337-342.

[20] N.K. Jayant, “Attendance Management System Using Hybrid Face Recognition Techniques," pp. 412-417, 2016.

[21] S.Kumar Rath and S. Swarup Rautaray, "A Survey on Face Detection and Recognition Techniques in Different Application Domain,” Int. J. Mod. Educ. Comput. Sci., vol. 6, no. 8, pp. 34-44, 2014.

[22] M. Sajid, R. Hussain and M. Usman, "A conceptual model for automated attendance marking system using facial recognition," Ninth International Conference on Digital Information Management (ICDIM 2014), Phitsanulok, 2014, pp. 7-10.

[23] J. Kaur and A. Sharma, "Performance analysis of face detection by using Viola-Jones algorithm," International Journal of Computational Intelligence Research. 2017, vol. 13, no. 5, pp. 707-717.

[24] K. Kadir, M. K. Kamaruddin, H. Nasir, S. I. Safie, and Z. A. K. Bakti, "A comparative study between LBP and Haar-like features for Face Detection using OpenCV,” 2014 4th Int. Conf. Eng. Technol. Technopreneuship, ICE2T 2014, vol. 2014-Augus, pp. 335-339, 2014.

[25] P. K. Dhere, "Review of PCA , LDA and LBP algorithms used for 3D Face Recognition," Int. J. Eng. Sci. Innov. Technol., vol. 4, no. 1, pp. 2008-2011, 2015. 